\title{
Análisis del estado del arte sobre el uso de los videojuegos en Educación Infantil y Primaria
}

\author{
Analysis of the state of the art on the use of video games \\ in Nursery and Primary Education
}

RECIBIDO 15/1/2021 ACEPTADO 10/5/2021 PUBLICADO 1/12/2021

\section{Diana Marín Suelves}

Departament de Didàctica i Organització Escolar, Universitat de València, España

diana.marin@uv.es

(D) María Isabel Vidal Esteve

Departament de Didàctica i Organització Escolar, Universitat de València, España

isabel.vidal@uv.es

(i) Donatella Donato

Departament de Teoria de l'Educació, Universitat de València, España

dodo2@uv.es

Jesús Granados Saiz

Departament de Didàctica i Organització Escolar, Universitat de València, España

jesusgranadossaiz@gmail.com

\section{RESUMEN}

La integración de las tecnologías en el ámbito educativo es una realidad innegable en la sociedad del siglo XXI, caracterizada por los rápidos cambios que posibilitan la revolución educativa y la innovación. Muestra de ello es el uso de videojuegos en los centros escolares de las etapas de Educación Infantil y Primaria. Estas prácticas gamificadas mediante tecnología no están exentas de críticas o susceptibilidades sobre sus posibilidades educativas. Con el fin de describir los beneficios y posibilidades de la introducción de los videojuegos en las aulas detectados en la literatura científica, se ha realizado un estudio de revisión bibliográfica. Para ello, se utilizaron cuatro bases de datos (Scopus, WOS, ERIC y Dialnet) y se seleccionaron un total de 18 documentos para su análisis, siguiendo el método PRISMA. Los resultados muestran que los videojuegos pueden contribuir positivamente a los procesos de enseñanza-aprendizaje, desarrollar aspectos cognitivos y creativos e, incluso, a mejorar algunas actitudes sociales. En definitiva, este estudio permite evidenciar las posibilidades del uso educativo de los videojuegos con alumnado de Educación Infantil y Primaria, siempre y cuando se cuente con profesorado formado y competente digitalmente, y la selección del recurso se realice considerando la dimensión pedagógica, además de la técnica.

PALABRAS CLAVE infancia, tecnología, videojuegos, educación. 


\begin{abstract}
The integration of technologies in the educational field is an undeniable reality in the 21st century society, characterized by the rapid changes that make the educational revolution and innovation possible. Proof of this is the use of video games in schools in the Infant and Primary Education stages. These gamified practices through technology are not exempt from criticism or sensitivity about their educational possibilities. In order to describe the benefits and possibilities of the introduction of video games in classrooms detected in the scientific literature, a literature review study has been carried out. For this, the Scopus, WOS, ERIC and Dialnet databases were used, and a total of 18 documents were selected for analysis using the PRISMA method. The results show that video games can contribute positively to teaching-learning processes, develop cognitive and creative aspects, and even improve some social attitudes. In short, this study demonstrates the possibilities of the educational use of video games with students of Nursery and Primary Education, as long as there are digitally trained and competent teachers, and the selection of the resource is made considering the pedagogical dimension, in addition to the technique.
\end{abstract}

KEYWORDS childhood, technology, video games, education.

\title{
1. PLANTEAMIENTO DEL PROBLEMA
}

El impacto de las tecnologías en todos los ámbitos de la vida, y en concreto en la educación, es evidente. Las TIC, con formatos que abarcan desde la inteligencia artificial a la realidad virtual pasando por los videojuegos, y acompañadas de metodologías activas y novedosas, se están implementando en las escuelas de forma cada vez más extendida.

Así pues, esta investigación se centra en conocer los usos y los resultados que está aportando la introducción de una serie de elementos que forman parte de los diseños curriculares escolares y que tienen la virtud de mostrar universos complejos a través de un lenguaje que conversa directa e intensamente con la cultura visual de niños; los videojuegos (Esnaola, 2006). El número de artículos publicados sobre el uso de los videojuegos en el ámbito educativo se ha incrementado notablemente en los últimos años (Marín, et al., en prensa). Sin embargo, como se ha podido observar, la mayoría de los estudios se centran en Educación Secundaria o estudios superiores. Por este motivo, esta investigación se centra en analizar su influencia en las etapas de Educación Infantil y Primaria.

Bien es cierto que el uso de los videojuegos en el ámbito educativo está convirtiéndose en un tema controvertido que despierta argumentos y valoraciones tanto a favor como en contra. Es decir, se registran tanto beneficios en el aprendizaje, en el desarrollo de competencias (Holguín et al., 2020), en la motivación y creatividad (Hervás et al., 2018), o en el desarrollo de funciones socio-emocionales (Guerra et al., 2018), como riesgos en cuanto a aislamiento social, violencia, sedentarismo o adicción a su utilización (MartínezGonzález, 2020). Por este motivo, es interesante conocer en profundidad la evidencia científica publicada al respecto sobre su uso en aulas de Educación Infantil y Primaria.

Por último, se considera que la idea de introducir elementos innovadores en la escuela requiere de un proceso de evaluación y revisión constante. En este sentido, un análisis del estado del arte permitirá realizar una valoración global de lo que está suponiendo la introducción de los videojuegos en la escuela. 


\section{ANTECEDENTES Y FUNDAMENTACIÓN TEÓRICA}

Las sociedades actuales y las tendencias de ocio están jugando un papel cada vez más importante en el ámbito educativo. En estos últimos años. han alcanzado una cierta madurez para convertirse en una alternativa a los métodos tradicionales de aprendizaje. La integración de las tecnologías digitales en la educación a través de hardware, software, conexiones a Internet, contenido digital y formación para profesores no ha demostrado ser suficiente para alterar los métodos de enseñanza-aprendizaje y producir cambios que puedan considerarse una auténtica revolución a nivel global. Se trata de satisfacer esta necesidad de innovación y motivación a través de la gamificación en educación, ya que la vocación de un juego es hacer atractivo el aprendizaje mediante la introducción de escenarios e interacciones lúdicas.

El eje de la gamificación en educación lo constituyen los beneficios que aporta un diseño curricular basado en juegos, ayudando a mantener el interés de los alumnos y evitando que el proceso de enseñanzaaprendizaje se convierta en algo aburrido o sin interés para ellos, disminuyendo la falta de compromiso en el proceso de enseñanza y favoreciendo la adquisición de competencias (Area, \& González, 2015). Sin embargo, sería poco realista suponer que los juegos pueden proporcionar una solución inmediata y perfecta a los problemas actuales de innovación e interés del alumnado, aunque hay muchos defensores de la contribución y el potencial de los videojuegos como el papel que podrían desempeñar en la educación formal.

Adentrándonos en aspectos relevantes, la utilización de videojuegos con fines didácticos abarca tanto la metodología de aprendizaje como la motivación del alumnado independientemente de la materia que estén estudiando. En comparación con los medios tradicionales, tienen un ingrediente añadido: la interacción (Corsi et al., 2019). Sin embargo, no es tan innovador el efecto transformador de los videojuegos en el contexto educativo si se compara con el concepto de alfabetización digital según Torres-Toukoumidis et al. (2016).

El gran potencial de los juegos digitales o videojuegos radica en que sus atributos pueden integrarse en un proceso que se orienta hacia el aprendizaje. Los atributos (Cela et al., 2014), se traducen en determinadas posibilidades educativas de los mundos virtuales, que surgen de las teorías del aprendizaje relacionadas con el constructivismo, como la colaboración y compromiso activo, la construcción del conocimiento, la flexibilidad, la motivación, la competencia, la inmersión y la interacción con el medio ambiente. En palabras de Ibáñez et al. (2011), "los mundos virtuales abren la puerta a una nueva forma de aprender" (p. 8). La tendencia educativa y la incorporación de los videojuegos requieren hablar de su capacidad para enseñar contenidos y desarrollar habilidades. Su introducción en el aula implica un aumento de la motivación para el aprendizaje, ya que un entorno real, como el del espacio de juego informal de cada alumno, es decir, referido a su tiempo libre, pasa a uno formal de aprendizaje.

El principal desafío es examinar los beneficios y posibilidades de la introducción de los videojuegos en las aulas, en los aspectos educativos, técnicos y lúdicos de los juegos para determinar los criterios de selección y uso la educación formal. A través de este trabajo, se pretende hacer una contribución en esta área a partir del análisis de diferentes estudios para conocer el estado del arte. La originalidad del trabajo en cuestión radica en el hecho de que se analiza y explora el uso de los videojuegos en las aulas de Infantil y Primaria. Aunque la principal preocupación sigue siendo la eficacia, se acentúa cada vez más que el alumnado 
se relacione de forma diaria con elementos tecnológicos, así como el sinfín de softwares y apps digitales, desarrollando su competencia digital a la vez que se produce el aprendizaje. Por lo tanto, con este trabajo se busca responder a cuestiones como: ¿existe un uso extendido de los videojuegos en educación?, ¿están los niños de infantil y primaria preparados para aprender a través de los videojuegos?, ¿qué posibilidades tiene el uso de videojuegos?, ¿qué implicaciones tienen los videojuegos en el aprendizaje? o ¿qué características tienen los videojuegos empleados en las aulas?

\section{DISEÑO Y METODOLOGÍA}

Este trabajo es un estudio sobre el estado del arte (Vélez, \& Galeano, 2002), es decir, la situación de conocimiento de la comunidad científica sobre el uso educativo de los videojuegos. El análisis del estado del arte es una forma de publicación secundaria, mediante la cual es posible resumir los resultados conseguidos en otros estudios, relacionando los datos y sacando conclusiones, con el fin de generar nuevo conocimiento sobre un tema específico. Tal y como apunta Molina (2005), se trata de un estudio analítico de los distintos saberes escritos acumulados sobre un ámbito concreto. Gracias al análisis crítico y detallado de la literatura publicada, el trabajo de revisión desempeña un papel importante en la definición, descripción y estructuración de un cuadro completo del problema examinado, teniendo en cuenta, a su vez, la especificidad y el contexto en el que se desarrolla cada investigación (Icart, \& Canela, 1994); además permite descubrir conocimientos actuales que conducen a la confirmación, desarrollo o refinamiento de teorías, así como a plantear nuevas preguntas sobre la investigación (Trinchero, 2002).

En esta línea, este artículo se basa en la revisión sistemática de publicaciones realizadas a nivel nacional e internacional, en los últimos diez años, para encontrar la relación entre los videojuegos y los procesos de enseñanza-aprendizaje en Educación Infantil y Primaria. La recogida de información se llevó a cabo en las bases de datos WOS, SCOPUS, ERIC y Dialnet, por la cantidad y calidad de resultados de revistas científicas en el campo de las Ciencias Sociales. Para la búsqueda se emplearon como palabras clave: videogame OR serious game AND education AND nursery OR primary school, en castellano e inglés. Se establecieron como criterios de inclusión: artículos científicos, publicados en la última década, disponibles en abierto. En la selección de los artículos, no se tuvieron restricciones de tipo geográfico y/o cultural. Respecto a los criterios de exclusión, no se consideraron los estudios con muestra no incluida en la etapa educativa de Educación Infantil o Educación Primaria, aquellos que no utilizaban videojuegos en el aula, ni revisiones sistemáticas. Se eliminaron todos aquellos que no se ajustaban al objetivo del estudio y los repetidos. En definitiva, se han incluido en este estudio un total de 18 artículos, procediendo un $16,7 \%$ de la base de datos Wos, $16,7 \%$ de ERIC, 5,6\% de Dialnet y un 44,4\% de la base de datos Scopus y el resto emergen de la búsqueda inversa (16,7\%).

El proceso de selección de los estudios mediante el método PRISMA (Urrútia, \& Bonfill, 2010), se ilustra en el siguiente diagrama de flujo (Figura 1). 
Figura 1. Proceso de selección de documentos. Fuente: elaboración propia.

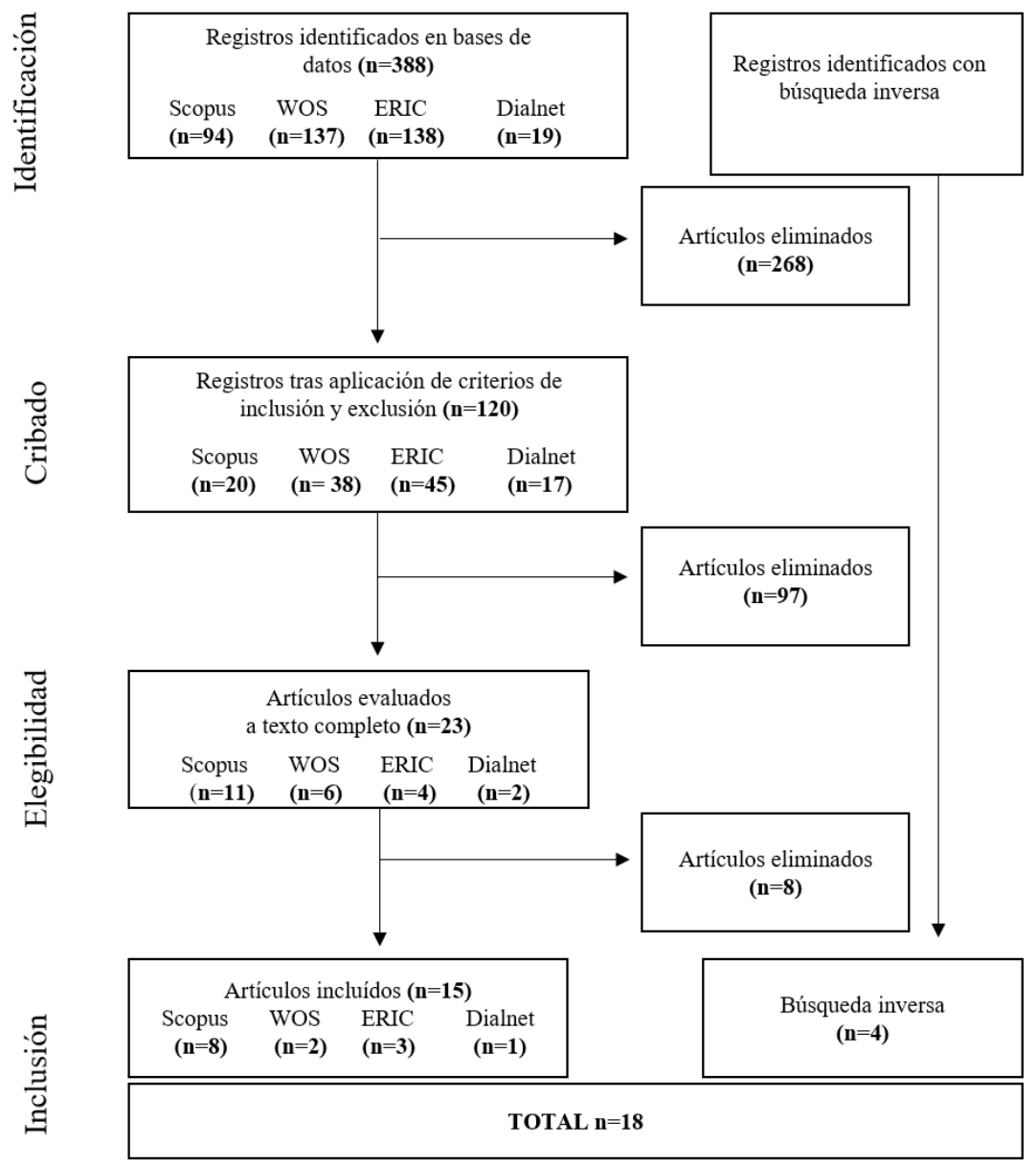

Una vez seleccionados los documentos a incluir en el análisis de contenido, y tras la lectura independiente de cada uno de ellos por parte de dos de las investigadoras autoras del artículo, se concretaron las siguientes dimensiones: la autoría y fecha de publicación, los objetivos perseguidos, la muestra participante, el videojuego utilizado y los resultados alcanzados.

\section{RESULTADOS}

En la Tabla 1, se muestran los rasgos principales de cada uno de los documentos analizados de acuerdo a las categorías establecidas. 
TABLA 1. Principales rasgos de los artículos analizados.

\begin{tabular}{|c|c|}
\hline AUTORÍA Y FECHA & VIDEOJUEGO, OBJETIVOS Y MUESTRA \\
\hline \multirow{7}{*}{$\begin{array}{l}\text { Garmen, Rodríguez, } \\
\text { García-Redondo, \& } \\
\text { San Pedro, } 2019\end{array}$} & Software TOI \\
\hline & Trabajar las inteligencias: lingüística, lógico-ma- \\
\hline & \\
\hline & intrapersonal, interpersonal y naturalista. \\
\hline & $n=372$ alumnos de tres centros de E.P. de Asturias y \\
\hline & Madrid. \\
\hline & Edades entre 5 y 9 años. \\
\hline
\end{tabular}

No existen diferencias significativas por género, pero sí por curso y edad de los participantes.

TOI se considera un instrumento adecuado para evaluar e intervenir las Inteligencias Múltiples.

Su inclusión en el aula tiene importantes implicaciones educativas dando valor a la comunidad educativa.

Permite conocer el perfil del alumnado, y adaptar la enseñanza al estilo de aprendizaje para una educación más personalizada e inclusiva.

\begin{tabular}{lll}
\hline $\begin{array}{l}\text { Tazouti, Boulakna- } \\
\text { del, \& Fakhri, } 2019\end{array}$ & JeuTICE & Alta satisfacción de los participantes. \\
& Desarrollar la competencia matemática. & Aumenta el interés y el compromiso del estudian- \\
& Edades de 10 a 11 años. & te para resolver los problemas y su confianza. \\
& & Mejora el aprendizaje desarrollando las habilida- \\
& des básicas de las matemáticas y las informáticas.
\end{tabular}

\section{Hawkins, Mendes, \& Farm Animal Welfare}

Williams, 2019
Se pretende diseñar y evaluar un juego digital para enseñar a los niños sobre el bienestar de los animales de granja.

$\mathrm{n}=133$ alumnos $\mathrm{E}$. P. $(\mathrm{GE}=69, \mathrm{GC}=64)$.

Edades entre 6 y 13 años.
Incremento en el conocimiento de los niños sobre el contexto rural, el bienestar animal, los sistemas de crías, etc. demostrando por tanto los beneficios de usar juegos serios en Educación Primaria.
González-Valero, Ubago-Jiménez, Zurita-Ortega, Chacón-Cuberos, Castro-Sánchez, \& Puertas-Molero, 2018

Castro, RaposoRivas, \& MartínezFigueira, 2018
Desarrollar hábitos alimenticios y un estilo de vida saludable.

Describir los niveles de masa corporal en relación con el uso problemático de los videojuegos.

n=577 alumnos de Educación Primaria.

Edades de 10 a 12 años.
Los sujetos con un uso excesivo de videojuegos muestran problemas de comunicación y comportamiento en el entorno escolar y un peor rendimiento académico.

La mitad de los participantes necesitan mejorar la adherencia a la dieta mediterránea.

\section{El Profesor Layton y la Villa Misteriosa}

Mejorar la atención.

$\mathrm{n}=6$ alumnos de quinto curso de Educación Primaria.
Aumenta la curiosidad científica y el trabajo individual y colaborativo.

Los participantes mostraron actitudes positivas hacia las propuestas, especialmente referidas a lo audiovisual, los personajes y el hilo argumental. Mejora la cognición y la motivación.

Las características del videojuego permiten su uso para mejorar la atención de alumnado con NEAE, al igual que el resto de los usuarios.
Strawhacker, Bers, Verish, Sullivan, \& Shaer, 2018

\section{BacToMars videogame}

Adquirir conceptos y entender la naturaleza interdisciplinaria de la ciencia.

n= 17 de E. P., divididos en grupo de intervención y control.

Edades de 7 a 11 años.
Los niños que usaron BacToMars mostraron mejor rendimiento en la comprensión de conceptos específicos que aparecen en el juego.

Este videojuego permite contextualizar conceptos complejos sobre ciencia e ingeniería.

Mejora la implicación del alumnado en la resolución de problemas interdisciplinarios de la vida cotidiana. 


Al Saud, 2017 Explorar los efectos de una selección de 10 video-
juegos educativos para iPad (descargados de http://
www.al3ab5l5l.com/learning-games.html) en el
enriquecimiento de habilidades sociales en niños
de jardín de infancia desfavorecidos.
n= 20 niños de 5 años.
Gl: 10 con acceso a videojuegos y al iPad.
GC: 10 sin acceso.

Puspitasari, 2017 Juego educativo en 2D para Android que parte del método Belajar Membaca Tanpa Mengeja.

Diseñar y desarrollar un juego educativo como herramienta efectiva para el aprendizaje de la lectura en la infancia.

$\mathrm{n}=26$ alumnos de $\mathrm{E}$. Infantil divididos en $\mathrm{GE}=13$ que usó la App y GC=13 que empleó un libro de lectura.

\section{Jenson, De Cas-} tell, Muehrer, \& McLaughlin-Jenkins, 2016

\section{Compareware}

Diseñar e implementar un videojuego educativo en estudiantes de $1^{\mathrm{a}}$ y $2^{\mathrm{a}}$ curso de primaria disponible para PC y iPad.

$\mathrm{n}=146$ alumnos de un total de 4 colegios de entre 6-8 años ( $1^{\circ}$ y $2^{\circ}$ curso).
Los niños desfavorecidos se volvieron más sociables y usaban expresiones como "adiós, gracias, disculpe, lo siento".
El grupo experimental tuvo una mayor puntuación post-test que el grupo de control.

Mejora la capacidad de lectura y puede ser usado como herramienta alternativa al libro para aprender a leer.
Cuando los alumnos obtenían feedback se sentían más motivados y comprometidos con la tarea.

\section{Lunn, Khalaf, Husain, Ancient Egypcians} Al-Jumeily, Pich, \& McCarthy, 2016
Este estudio de caso pretende implementar un juego serio basado en el Aprendizaje Abierto, diseñado para trabajar historia en niños de 7-8 años, captando al máximo su atención e involucrándose en su uso.

$\mathrm{n}=$ No especificada (niños de 7-8 años)
Los niños disfrutan y aprenden usando juegos serios, en varios entornos de aprendizaje, lo que amplía su potencial educativo no solo en las escuelas, sino también en otros entornos. El Aprendizaje Abierto puede ser una forma efectiva de motivar e involucrar a los niños para lograr sus metas de aprendizaje. Proporciona retroalimentación inmediata, motivación, entusiasmo y entretenimiento.

La práctica integrada por el folklore y los videojuegos favorece el aprendizaje eficiente de los alumnos en el ámbito del folklore (colocar notas en el pentagrama, diferenciar una jota de una sevillana, identificar una muñeira y recordar una canción popular al jugar con el videojuego).
Ramos, \& Botella, 2015 $\mathrm{n}=46$ alumnos de primero y segundo de educación primaria.

Aplicar el videojuego en primero y segundo de primaria para recuperar la tradición popular del pasado y proyectarla hacia las nuevas generaciones para familiarizarlas con sus raíces folklóricas.

Mozart en La Flauta Mágica mediante el editor de videojuegos E-adventure.

- Utilizar los videojuegos en el aula de Música.

- Iniciar a los alumnos en la percepción de la ópera a través de los videojuegos.

- Motivarles, haciéndoles partícipes de su propio aprendizaje.

- Fomentar el respeto, la cooperación y el uso responsable y coherente de los videojuegos.
El uso de videojuegos para acercar la ópera a los alumnos ha generado experiencias de aprendizaje que permiten el desarrollo personal, autonomía, y el descubrimiento, sin ejercer ningún tipo de impacto negativo o nocivo al usuario como la violencia o adicción. 
Zhao, \& Linaza Iglesias, 2015

\section{Xbox360: game Skylanders}

Conocer el impacto del uso de videojuegos en el aprendizaje y desarrollo de niños y niñas en edad escolar.

n=50 niños en grupos de 4 jugadores, 2 niños y 2 niñas por grupo.

De $2^{\mathrm{a}}, 4^{\mathrm{a}}$ y $6^{\mathrm{a}}$ grado de Educación Primaria.
Destacan las posibilidades de esta herramienta para el aprendizaje.

Desarrolla la coordinación y cooperación entre los miembros del equipo para lograr el aprendizaje esperado.

Se alcanzan mayores niveles de autonomía, resolución de conflictos y autorregulación para el juego.

El interés del alumnado por utilizar el juego fue elevado, tanto niños como niñas disfrutaron de la experiencia y aprendieron igual de rápido, independientemente del grupo de edad. Los alumnos asumen diferentes roles, juegan juntos, a pesar de la preferencia por jugar con personas de su mismo género y entienden los controles básicos.

Ayudan a sus compañeros a ser más competentes y favorece la socialización.

Mejora del proceso de enseñanza aprendizaje, aumento de la autonomía del alumnado facilitando su desarrollo motor (coordinación, motricidad y organización espaciotemporal), cognitivo (resolución de problemas, atención, curiosidad y tecnología) y socioafectivo (integración, motivación, autoestima y colaboración).

No se aprecian mejoras significativas en los participantes y los padres mantienen valoraciones negativas.

Los participantes consideran que Minecraft Edu es divertido (98.1\%), mejora la creatividad (91.4\%), se descubren cosas nuevas (77.9\%) y se aprende (74.1\%). Además, aplica de un modo efectivo contenidos orientados a edificios históricos (97.1\%) y permite trabajar interactuando con grupos de otros países siendo enriquecedor e interesante (93.3\%).

La mitad de los alumnos responden negativamente en cuanto a su utilidad y adecuación en el aula: se aprovecha el tiempo al utilizar los juegos serios (49\%) y es apropiado utilizarlos en clase (47.1\%).

Aumento de los conocimientos sobre nutrición.

Alta aceptación y usabilidad de la herramienta informada por los participantes.

Valoran la diversión y el entretenimiento frente a la información escrita.

Es un juego accesible a todos. 


$\begin{array}{ll}\text { Anyaegbu, Ting, \& } & \text { Mingoville } \\ \text { Li, 2012 } & \text { Analizar cómo Mingoville motivaría, involucraría y } \\ & \text { despertaría el interés de los estudiantes que preten- } \\ & \text { den dominar el inglés como lengua extranjera. } \\ & \mathrm{n}=229 \text { estudiantes de entre } 8 \text { y } 11 \text { años de dos escue- } \\ & \text { las de Primaria de Nanjing (China). }\end{array}$

las de Primaria de Nanjing (China).
Se identifican diferencias sobre cómo Mingoville motivó a los estudiantes en las dos escuelas; los factores condicionantes fueron la diferencia de dotación tecnológica (brecha digital) entre los estudiantes de ambos centros, la ratio de cada aula (recomiendan entre 10-15 alumnos para implementar adecuadamente videojuegos), la actitud del profesorado (había algunos reacios o con pocas habilidades para el manejo), el nivel de autonomía o de control que ejercía el docente ante el uso del videojuego.

Mejora la organización de actividades de enseñanza y aprendizaje del inglés para los/as principiantes. Los usuarios pueden comprometerse con Mingoville "sólo por diversión", mientras que el profesorado puede utilizar Mingoville para la enseñanza comprometida del inglés.

En cuanto a las características de los juegos utilizados, el panorama es bastante amplio y abarca juegos de simulación, juegos de rol, juegos de aventuras y juegos de lógica. También se contemplan diferentes estrategias, entre ellas: la exploración, la libre navegación, preguntas y respuestas, y la construcción de entornos, caminos u objetos. Los juegos empleados en los artículos analizados pueden ser tanto educativos, es decir, creados específicamente para cumplir objetivos educativos, como juegos creados con una intención exclusivamente lúdica que se utilizan para actividades de aprendizaje o para la mejora de habilidades específicas.

Al identificar el potencial de los videojuegos con fines educativos, la mayoría de los estudios destacan sus papeles en la motivación de los estudiantes y en el desarrollo de las habilidades de exploración, imaginación y reflexión.

\section{DISCUSIÓN}

La discusión de los resultados se organiza en base a variables estructurales, como la fecha, lugar de las publicaciones e instituciones de origen; variables metodológicas como las características de la muestra; y variables de contenido, como los objetivos que perseguía cada intervención y los resultados alcanzados.

En primer lugar, en cuanto a las variables estructurales, en la distribución temporal de las publicaciones destaca la existencia de una mayor cantidad de documentos publicados en los últimos años. A pesar de existir experiencias de aplicación de videojuegos en Infantil y Primaria en la última década, es desde hace un lustro cuando el número de publicaciones ha crecido, representando un $67 \%$ del total de documentos analizados. Por lo que respecta al origen de las publicaciones, resalta el peso de países como España (50\%), seguido de Reino Unido (11,1\%) y otros países como Dinamarca, China, Irak, Arabia Saudita, Canadá, Indonesia, Marruecos y USA, que cuentan con un artículo cada uno. Por lo que se refiere a la colaboración, medida a través del grado de colaboración entre autores, destaca que un $44,4 \%$ de los artículos analizados son firmados por in- 
vestigadores pertenecientes a la misma institución, otro 44,4\% en el que los autores son de distintas universidades, pero situadas en un mismo país, mientras que, tan sólo en un caso los autores pertenecen a países distintos - Reino Unido e Irak - en Lunn et al. (2016).

En segundo lugar, respecto a las variables metodológicas, es necesario señalar las grandes diferencias entre los tamaños muestrales utilizados y la existencia o no de grupo de control.

Cabe destacar también que la mayoría de las intervenciones analizadas emplean videojuegos en la etapa de Educación Primaria, mientras que tan sólo un 5,5\% de los estudios implican a participantes pertenecientes a la etapa de Educación Infantil. Esto podría deberse a las características psicoevolutivas del alumnado, ya que no es hasta los 6 años cuando la maduración de sus facultades cognitivas y sensomotoras está prácticamente completa (Martín, 2008), o bien, a las metodologías implementadas en dicha etapa, de cariz más naturalista, activo y vivencial. Por lo que respecta a las edades de los participantes, este estudio muestra como es más frecuente la implementación de los videojuegos en el ámbito educativo formal, con alumnado de los últimos cursos de Educación Primaria.

En tercer lugar, es posible diferenciar los objetivos de las intervenciones en cuatro bloques. Mientras unos se dirigen al desarrollo de habilidades cognitivas como la atención (Castro et al., 2018), las habilidades sociales (Al Saud, 2017) o las inteligencias múltiples (Garmen et al., 2019), otros pretenden mejorar el aprendizaje de áreas específicas como las Ciencias Naturales (Hurtado et al., 2015; Strawhacker et al., 2018), las Matemáticas (Tazouti et al., 2019), la Lengua Inglesa (Anyaegbu et al., 2012; Hansbøl, \& Meyer, 2011), la Historia (Lunn et al., 2016) o la Educación Musical, trabajando la ópera (Ramos, \& Botella, 2015) o la recuperación de las raíces folklóricas (Ramos, \& Botella, 2016). El tercer grupo se centra en temas transversales, como la educación para la salud o la nutrición (Baños et al., 2013; González-Valero et al., 2018), o el cuidado de los animales y el fomento de su bienestar (Hawkins et al., 2019). Por último, destacan aquellos que lo que pretenden analizar es el impacto de los videojuegos en el alumnado (Jenson et al., 2016; Zhao, \& Linaza, 2015) o sus beneficios pedagógicos (Sáez-López, \& Domínguez-Garrido, 2014). Además, es de destacar que algunos de los artículos contemplan también como uno de sus objetivos específicos incrementar los niveles de motivación y compromiso del alumnado (Hurtado et al., 2015; Lunn et al., 2016; Sáez-López, \& Domínguez-Garrido, 2014).

En general, como en estudios previos, los resultados muestran la potencialidad de los videojuegos en el ámbito educativo y su utilidad para facilitar el aprendizaje desde una perspectiva multisensorial ( $\mathrm{Pa}$ panastasiou et al., 2017; Skianis, 2017), estimular el pensamiento cognitivo, creativo, social y moral del alumnado (Galindo, 2019), cambios en los comportamientos para promover estilos de vida saludables (Baños et al., 2013), y la transversalidad en los entornos educativos a través del desarrollo de la alfabetización mediática (Sousa, \& Cost, 2018), ya que constituyen una herramienta muy motivadora, porque el alumnado aprende jugando en un entorno desafiante (Papanastasiou et al., 2017). Así, se observa en trabajos como el de Ramos y Botella (2016) que el uso de estrategias didácticas novedosas y recursos tecnológicos de forma innovadora aumenta la participación del alumnado y convierte al docente en un facilitador del acceso al aprendizaje (Hurtado et al., 2015). Otros estudios (Anyaegbu et al., 2012) muestran que la actitud, tanto de los docentes como de las familias, es un factor clave para un aprendizaje exitoso basado en videojuegos. Muestra de ello, es la influencia del nivel socioeducativo de las familias en el consumo de pantallas inteligentes, encontrada por Jiménez-Morales et al. (2020). Además, Al Saud (2017) manifiesta que el emplear videojuegos permite mejorar las habilidades sociales de todo el alumnado y reducir tanto la brecha digital 
como la económica. Así pues, los resultados de este análisis del estado del arte coinciden con lo concluido por otros autores como Checa-Romero (2016), al confirmar el componente tanto educativo como lúdico de muchos de estos juegos y su potencial motivador (Barreto et al., 2017). Sin embargo, varias investigaciones señalan que el análisis de la eficacia de su uso debe examinarse en contextos específicos y de acuerdo con objetivos y situaciones claramente definidas. Por consiguiente, el profesorado debe permanecer informado del potencial, las características y las limitaciones de estos instrumentos, pero también debe saber seleccionar, desde un punto de vista pedagógico, juegos apropiados y diseñar y ejecutar actividades de aprendizaje ricas y bien estructuradas, teniendo en cuenta las necesidades tanto de la clase en su conjunto como del alumnado en particular (Lobel et al., 2017).

\section{CONCLUSIONES}

Los estudios de revisión de la literatura permiten obtener información a partir del análisis del estado del arte en un campo específico como, en este caso, sobre el uso e implicaciones de la introducción de los videojuegos en las aulas de Infantil y Primaria, lo que permite alcanzar conclusiones y realizar propuestas de futuro vinculando teoría y práctica con ciertas garantías de éxito.

El interés por el análisis del uso de los videojuegos en el campo educativo permite aproximarse a esta nueva generación de estudiantes que usarán la tecnología de forma habitual para comunicarse, expresarse y comprender el mundo que les rodea, y para dar muestras de las evidencias obtenidas y así determinar las posibilidades y riesgos que su uso en la escuela realmente puede acarrear.

Desde hace tiempo, el juego es considerado un aspecto muy importante del desarrollo individual y social del niño y, hoy en día, cuando se habla de juegos, no se pueden obviar los videojuegos, que representan la forma preferida y más utilizada por los adolescentes (Ricoy, \& Ameneiros, 2016). Los videojuegos se han ido abriendo camino también en la educación, no sin una cierta dificultad y resistencia (Fernández, \& Luque, 2021), principalmente debido a la falta de una formación adecuada por parte de toda la comunidad educativa. En los últimos años, se han realizado muchas investigaciones que han puesto de relieve el potencial educativo concreto de los juegos digitales, especialmente teniendo en cuenta el impacto positivo en la dimensión cognitiva, motivacional, emocional y social, y su uso en la escuela está empezando a ser bastante generalizado en muchos países (Collins, \& Halverson, 2014).

Junto con los escenarios atractivos, que cuanto más simulan la realidad, más eficaces son en términos de aprendizaje significativo, el videojuego en la educación podría ser un medio empático y contemporáneo para generar cultura inclusiva.

El juego, a diferencia de otros medios, nos permite convertirnos en protagonistas, identificarnos, abriendo la posibilidad de observar el mundo a través de segmentos de la realidad que de otro modo quedarían excluidos y permite también trabajar en la dimensión de la empatía, el cuidado, la consideración, el apoyo mutuo y el trabajo en equipo (Viola, \& Cassone, 2017). Además, los juegos enseñan a los niños tareas y habilidades complejas, permitiendo a los jugadores, siempre que el nivel de dificultad sea el adecuado y los objetivos coherentes y comprensibles, procesar mejor los aprendizajes y mantenerlos al límite de su capacidad, proporcionando mejoras en una amplia variedad de habilidades perceptivas, atencionales y cognitivas (Papanastasiou et al., 2017). 
Este es el desafío para los profesionales de la educación: ver en el uso de los videojuegos en el proceso de enseñanza-aprendizaje la posibilidad de entrenar el desarrollo de habilidades de amplio espectro, así como reflexionar sobre las situaciones de exclusión social explícita por motivos de etnia, género, orientación sexual o religiosa, discapacidad o edad y poner de manifiesto las dinámicas discriminatorias implícitas, favoreciendo la adopción de un comportamiento inclusivo, de amplia participación y de compromiso (Giaconi, \& Del Bianco, 2019). En esta línea, los videojuegos se caracterizan por combinar estímulos diversos: auditivos, táctiles, visuales e intelectuales, que favorecen la motivación de todos y todas para participar y aprender.

Es tarea de la comunidad educativa en su conjunto investigar el potencial pedagógico de las herramientas disponibles, para una formación integral, utilizando los videojuegos como una opción que puede enriquecer el entorno en el que interactúan cada día.

En definitiva, una vez más parece que la clave del cambio en la escuela, de la innovación y de la introducción de recursos educativos diversos está en la formación del profesorado, de ahí la importancia de la realización de estudios como el de Antequera et al. (2019), en el que se utilizaron los videojuegos para el aprendizaje de futuros docentes de Educación Infantil. Por tanto, de acuerdo con Ramos y Botella (2016), el docente desde la perspectiva de la investigación en acción, debe reflexionar desde su propia práctica al incluir herramientas innovadoras emergentes en el aula, porque los videojuegos, por sí mismos, no generan mejoras en los procesos educativos si no están acompañados de un trabajo coherente con el diseño curricular, teniendo en cuenta los objetivos de aprendizaje, el grupo de alumnos y en qué circunstancias se utilizará el juego, tal y como afirman Papanastasiou et al. (2017).

\section{LIMITACIONES Y PROSPECTIVA}

Por lo que respecta a las limitaciones, es necesario mencionar el número reducido de estudios centrados en las etapas de Educación Infantil y Primaria, frente a la gran cantidad de estudios encontrados en los que los participantes son alumnado de Educación Secundaria y Educación Superior (Marín et al., en prensa). Además, en la mayoría de los estudios se contemplan grupos de intervención reducidos, en algunos de ellos no se cuenta con grupo de control, se basan en experiencias puntuales con videojuegos y no se realiza un seguimiento para determinar si las variables desarrolladas se mantienen en el tiempo.

Por tanto, como líneas de investigación futura se plantea la necesidad de superar las anteriores limitaciones y centrar el foco en el modelo pedagógico subyacente y en el papel de los videojuegos para la consecución de la inclusión del alumnado con necesidades específicas de apoyo educativo.

\section{FINANCIACIÓN}

El presente trabajo surge del proyecto I+D+i denominado: "Los materiales didácticos digitales en la Educación Infantil. Análisis y propuestas para su uso en la escuela y en el hogar" (RTI2018-093397-B-I00) y del proyecto de tesis doctoral con referencia FPU17/000372 financiados ambos por el Ministerio de Ciencia, Innovación y Universidades. 


\section{REFERENCIAS}

Al Saud, A. F. (2017). Educational Video Games Enrich Underprivileged Children's Social Skills in Saudi Arabia. European Journal of Educational Sciences, 4(2), 32-47. http://dx.doi. org/10.19044/ejes.v4no2a3

Antequera, J. A., Guerra, J., Teixeira, M. R., Costa, J. M., \& del Águila, Y. (2019). Estudio de la orientación y la percepción espacial en alumnado del Grado de Educación Infantil a través del portal 2. Revista INFAD de Psicología. International Journal of Developmental and Educational Psychology, 3(1), 563-576. https://doi.org/10.17060/ijodaep.2019.n1.v3.1536

Anyaegbu, R., Ting, W., \& Li, Y., (2012). Serious game motivation in an EFL classroom in Chinese primary school. TOJET: The Turkish Online Journal of Educational Technology, 11(1), 154-164.

Area, M., \& González, C. (2015). De la enseñanza con libros de texto al aprendizaje en espacios online gamificados. Educatio Siglo XXI, 33(3), 15-38. http://dx.doi.org/10.6018/j/240791

Baños, R. M., Cebolla, A., Oliver, E., Alcañiz, M., \& Botella, C. (2013). Efficacy and acceptability of an Internet platform to improve the learning of nutritional knowledge in children: the ETIOBE mates. Health education research, 28(2), 234-248. https://doi. org/10.1093/her/cys044

Barreto, D., Vasconcelos, L., \& Orey, M. (2017). Motivation and learning engagement through playing math video games. Malaysian Journal of Learning and Instruction, 14(2), 1-21.

Castro, Á. P., Raposo-Rivas, M., \& Martínez-Figueira, M. (2018). ¿Mejorar la atención con videojuegos? un estudio de caso. REOPRevista Española de Orientación y Psicopedagogía, 29(3), 94109. https://doi.org/10.5944/reop.vol.29.num.3.2018.23323

Cela, J. M., Esteve, F. M., Esteve, V., \& Gisbert, M. (2014). Developing self-management and teamwork using digital games in 3D simulations. Australasian Journal of Educational Technology, 30(6), 634-651. https://doi.org/doi:10.14742/ajet.754

Checa-Romero, M. (2016). Developing skills in digital contexts: Video games and films as learning tools at primary school. Games and Culture, 11(5), 463-488. https://doi. org/10.1177/1555412015569248
Collins, A., \& Halverson, R. (2014). La seconda rivoluzione educativa: come la tecnologia sta trasformando l'educazione. Italian Journal of Educational Technology, 22(1), 4-10.

Corsi, D., Revuelta, F. I., \& Pedrera, M. I. (2019). Adquisición de competencias emocionales mediante el desarrollo y uso de Serious Games en Educación Superior. Pixel-Bit: Revista de Medios y Educación, (56), 95-112. https://doi.org/10.12795/ pixelbit.2019.i56.05

Esnaola, G. A. (2006). Claves culturales en la construcción del conocimiento: ¿qué enseñan los videojuegos? Alfagrama Ediciones.

Fernandez, A. J., \& Luque, E. (2021). I Libro Blanco español de la I+ $D+$ iy Ciencia en videojuegos. UMA Editorial.

Galindo, H. (2019). Los videojuegos en el desarrollo multidisciplinar del currículo de Educación Primaria: el caso Minecraft. Pixel-Bit: Revista de Medios y Educación, (55), 57-73. https:// doi.org/10.12795/pixelbit. 2019.i55.04

Garmen, P., Rodríguez, C., García-Redondo, P., \& San Pedro, J. C. (2019). Multiple intelligences and video games: Assessment and intervention with TOI software. Comunicar. Media Education Research Journal, 27(1), 95-104. https://doi.org/10.3916/ C58-2019-09

Giaconi, C., \& Del Bianco, N. (2019). In azione: Prove di inclusione. FrancoAngeli.

González-Valero, G., Ubago-Jiménez, J. L., Zurita-Ortega, F., Chacón-Cuberos, R., Castro-Sánchez, M., \& Puertas-Molero, P. (2018). Eating Habits and Lifestyles in Schoolchildren from Granada (Spain). A Pilot Study. Education Sciences, 8(4), 1-10. https://doi.org/10.3390/educsci8040216

Guerra, J., Pedrera, M. I., \& Revuelta, F. I. (2018). Perception of teaching skills through emotions by the use of social theme video games. EDMETIC, 7(2), 57-77. https://doi.org/10.21071/ edmetic.v7i2.10950

Hansbøl, M., \& Meyer, B. (2011). Shifting ontologies of a serious game and its relationships with English education for beginners. E-learning and Digital Media, 8(3), 228-246. 
Hawkins, R. D., Mendes, G. A., \& Williams, J. M. (2019). The Development and Evaluation of 'Farm Animal Welfare': An Educational Computer Game for Children. Animals, 9(3), 1-17. https:// doi.org/10.3390/ani9030091

Hervás-Gómez, C., Ballesteros-Regaña, C., \& Corujo-Vélez, C. (2018). La robótica como estrategia didáctica para las aulas de Educación Primaria. Hekademos: revista educativa digital, (24), 30-40.

Holguin, J., Taxa, F., Flores, R., \& Olaya, S. (2020). Proyectos educativos de gamificación por videojuegos: desarrollo del pensamiento numérico y razonamiento escolar en contextos vulnerables. EDMETIC, Revista de Educación Mediática y TIC, 9(1), 80-103 https://doi.org/10.21071/edmetic.v9i1.12222

Hurtado, A., Ramírez, V., Talavera, M., \& Cantó, J. (2015). Aplicaciones educativas de los videojuegos: Una propuesta didáctica con Minecraft para el aula de ciencias. Revista Internacional de Aprendizaje y Cibersociedad, 19(1), 73-90.

Ibáñez, M.B., García, J.J., Galán, S., Maroto, D., Morillo, D., \& Kloos, CD (2011). Diseño y implementación de un mundo virtual multiusuario 3D para el aprendizaje de idiomas. Tecnología y sociedad educativas, 14(4), 2-10.

Icart, M.T., \& Canela, J. (1994). El artículo de revisión. Enfermería clínica, 4(4), 180-184.

Jenson, J., de Castell, S., Muehrer, R., \& McLaughlin-Jenkins, E. (2016). Playing and Learning: An iPad Game Development \& Implementation Case Study Jouer et apprendre: une étude de cas du développement et de la mise en œuvre d'un jeu sur iPad. Canadian Journal of Learning and Technology/La revue canadienne de l'apprentissage et de la technologie, 42(3). https://www.learntechlib.org/p/173433/

Jiménez-Morales, M., Montaña, M., \& Medina-Bravo, P. (2020). Childhood use of mobile devices: Influence of mothers' socio-educational level. Comunicar, 28(64), 21-28. https://doi. org/10.3916/C64-2020-02

Lobel, A., Engels, R. C., Stone, L. L., Burk, W. J., \& Granic, I. (2017) Video gaming and children's psychosocial wellbeing: A longitudinal study. Journal of youth and adolescence, 46(4), 884897. https://doi.org/10.1007/s10964-017-0646-z
Lunn, J., Khalaf, M., Hussain, A. J., Al-Jumeily, D., Pich, A., \& McCarthy, S. (2016). The use of serious gaming for open learning environments. Knowledge Management \& E-Learning: An International Journal, 8(1), 39-54. https://doi.org/10.34105/j. kmel.2016.08.004

Marín, D., Esnaola, G. \& Donato, D. (En prensa). Videojuegos y educación: análisis de tendencias en investigación. Revista Colombiana de Educación.

Martín, D. (2008). Psicomotricidad e intervención educativa. Ediciones Pirámide.

Martínez-González, J. S. (2020). Los videojuegos, una adicción para los adolescentes. Con-Ciencia Serrana Boletín Científico de la Escuela Preparatoria Ixtlahuaco, 2(3), 40-41.

Molina, N. P. (2005). ¿Qué es el estado del arte? Ciencia y Tecnología para la salud Visual y Ocular, 3(5), 73-75.

Papanastasiou, G., Drigas, A., \& Skianis, C. (2017). Serious games in preschool and primary education: benefits and impacts on curriculum course syllabus. International Journal of Emerging Technologies in Learning, 12(01), 44-56. https://doi. org/10.3991/ijet. v12i01.6065

Puspitasari, C. (2017). A New Tool to Facilitate Learning Reading for Early Childhood. Malaysian Online Journal of Educational Technology, 5(3), 1-15.

Ramos, S., \& Botella, A. M. (2015). Los videojuegos como herramientas de aprendizaje una experiencia de innovación con la ópera de Mozart. DEDICA: Revista de Educação e humanidades, 9, 161-171.

Ramos, S., \& Botella, A. M. (2016). La integración del videojuego educativo con el folklore. Una propuesta de aplicación en Educación Primaria. Revista Electrónica Interuniversitaria De Formación Del Profesorado, 19(3), 115-121. https://doi. org/10.6018/reifop.19.3.267281

Ricoy, C., \& Ameneiros, A. (2016). Preferencias, dedicación y problemáticas generadas por los videojuegos: Una perspectiva de género. Revista Complutense de Educación, 27(3), 12911308. https://doi.org/10.5209/rev RCED.2016.v27.n3.48445

Sáez-López, J.M., \& Domínguez-Garrido, C. (2014). Integración pedagógica de la aplicación minecraft edu en educación 
primaria: Un estudio de caso. Pixel-Bit. Revista de Medios y Educación, (45), 95-110. https://www.redalyc.org/articulo. oa?id=36831300007

Sousa, C., \& Costa, C. (2018). Videogames as a learning tool: is game-based learning more effective? Revista Lusófona de Educação 40(40), 199-210. https://doi.org/10.24140/issn.16457250.rle40.13

Strawhacker, A., Bers, M., Verish, C., Sullivan, A., \& Shaer, O. (2018). Enhancing children's interest and knowledge in bioengineering through an interactive videogame. Journal of Information Technology Education: Innovations in Practice, 17(1), 55-81. https://doi.org/10.28945/3976

Tazouti, Y., Boulaknadel, S., \& Fakhri, Y. (2019). JeuTICE: An Arabic Serious Game to Enhance Mathematics Skills of Young Children. International Journal of Emerging Technologies in Learning (iJET), 14(22), 252-265. https://doi.org/10.3991/ijet. $\underline{\text { v14i22.11119 }}$

Torres-Toukoumidis, A., Romero-Rodriguez, L., Amor, M., \& Bjork, S. (2016). Development of reading skills through video games: state of the art. OCNOS-Revista de estudios sobre la lectura, 15(2), 37-49. https://doi.org/10.18239/ocnos 2016.15.2.1124 Trinchero, R. (2002). Manuale di ricerca educativa. Franco Angeli. Urrútia, G., \& Bonfill, X. (2010). Declaración PRISMA: una propuesta para mejorar la publicación de revisiones sistemáticas y metaanálisis. Medicina clínica, 135(11), 507-511. https://doi. org/10.1016/j.medcli.2010.01.015

Vélez, O., \& Galeano, M. E. (Eds.) (2002). Investigación cualitativa: estado del arte. Centro de Investigaciones Sociales y Humanas.

Viola, F., \& Cassone, V. I. (2017). L'arte del coinvolgimento: emozioni e stimoli per cambiare il mondo. Hoepli editore.

Zhao, Z., \& Linaza, J. L. (2015). Relevance of videogames en the learning and development of young children. Electronic Journal of Research in Educational Psychology, 13(2), 301-318. https://doi.org/10.14204/ejrep.36.14108 